\title{
On the existence of solution in the linear elasticity with surface stresses
}

\author{
Holm Altenbach ${ }^{1, *}$, Victor A. Eremeyev ${ }^{2, * *}$, and Leonid P. Lebedev ${ }^{3, * * *}$ \\ ${ }^{1}$ Lehrstuhl für Technische Mechanik, Zentrum für Ingenieurwissenschaften, Martin-Luther-Universität Halle-Wittenberg, \\ 06099 Halle (Saale), Germany \\ 2 South Scientific Center of RASci \& South Federal University, Milchakova St. 8a, 344090 Rostov on Don, Russia \\ ${ }^{3}$ Universidad Nacional de Colombia, Cr. 45, \# 26-85, Bogotá D.C., Colombia
}

Key words Surface stresses, existence theorem, Sobolev's spaces, eigenfrequencies.

The mathematical investigation of the initial-boundary and boundary value problems in the linear elasticity considering surface stresses is presented. Weak setup of the problems based on mechanical variational principles is studied. Theorems of uniqueness and existence of the weak solution in energy spaces of static and dynamic problems are formulated and proved. Some properties of the spectrum of the problems under consideration are established. The studies are performed applying the functional analysis techniques. Finally, the Rayleigh principle for eigenfrequencies is constructed.

\section{Introduction}

The theory of elasticity that takes into consideration the action of stresses on the surface of a body is recently applied to problems of nanotechnology, cf. [4,5] among others. The surface stresses are supposed to be reliable for abnormal properties of nano-samples in comparison with macro-samples made of the same material. In particular, the surface stresses are responsible for the size-effect, that means the material properties of a specimen depend on its size. For example, Young's modulus of a cylindrical specimen increases significantly, when the cylinder diameter becomes very small. Together with the ordinary stresses $\sigma$ distributed in the volume the below presented theory takes into account independent surface stresses $\tau$ on the boundary that act similarly to the surface tension known in fluid mechanics [10]; $\tau$ depends on the surface deformation of the solid body.

The studies of the surface stresses in solids were initiated by J. W. Gibbs [7] at the end of XIXth century. The nonlinear mechanics with regard of surface stresses is developed in [8,9,15-18]. The model proposed in [8] can be considered as a model of an elastic body with an elastic film-membrane glued to its surface. In [18] this model is generalized to the surface films possessing bending stiffness. An important case of the theory for small deformations is presented in $[8,9,16,17]$ where the form of the constitutive equations is discussed. In [16] the constitutive relations for the moment interactions of the surface film with the body are proposed. A uniqueness theorem for linearly elastic body with surface stresses is proved in $[8]$.

The setup of the problem for linear elastic bodies with surface stresses consists of three dynamic or static differential equations of the second order in displacements. They are supplemented by boundary conditions that also contain the derivatives of the second order of the displacements in the tangent surface.

The following boundary value problem for Poisson's equation that describes a membrane illustrates the problems we meet in the theory of elastic bodies with surface stresses

$$
\nabla^{2} u=f, \quad(x, y) \in V,\left.\quad u\right|_{\Omega_{1}}=0,\left.\quad\left(\frac{\partial u}{\partial n}-\alpha \frac{\partial^{2} u}{\partial s^{2}}\right)\right|_{\Omega_{2}}=\varphi,
$$

where $u=u(x, y)$ is an unknown transversal deflection of the membrane, $f$ is an external load given on a bounded domain $V \subset \mathbb{R}^{2}$ and $\varphi$ is a load given on the part $\Omega_{2}$ of the boundary contour $\Omega$ of $V, \Omega_{1}=\Omega \backslash \Omega_{2}$. The $\partial / \partial n$ and $\partial / \partial s$ denote the directional derivatives along the normal and tangent directions to $\Omega$, respectively. 
Let $C_{0}^{(2)}$ be the set of all functions from $C^{(2)}(\bar{V})$ taking zero value on $\bar{\Omega}_{1}$. Problem (1) is equivalent to the following variational problem:

Given $f, \varphi$, find $u \in C_{0}^{(2)}$ that minimizes the functional

$$
J(u)=E(u)-A(u)
$$

on $C_{0}^{(2)}$. Here

$$
E(u)=\frac{1}{2} \int_{V}(\nabla u)^{2} d V+\frac{1}{2} \int_{\Omega_{2}} \alpha\left(\frac{\partial u}{\partial s}\right)^{2} d \Omega \quad \text { and } \quad A(u)=\int_{V} f u d V+\int_{\Omega} \varphi u d \Omega .
$$

$J(u)$ is the total energy functional for the membrane. The first integral in $E(u)$ corresponds to the "volume strain energy" and the second integral corresponds to the "surface strain energy" in the problems for an elastic body with surface stresses that will be considered in what follows. As for the strain energy for an elastic body with surface stresses, we need the $E$ to be non-negative. For the membrane problem this implies that the "module" $\alpha$ must be non-negative that we demonstrate with the following example.

Let $V=\{(x, y) \in[0,1] \times[0,1]\}, u=0$ on three square sides $\Omega_{1}=([0,1] \times\{0\}) \cup(\{0\} \times[0,1]) \cup(\{1\} \times[0,1])$ and $\alpha<0$. We show that the value of $E(u)$ that is now

$$
2 E(u)=\int_{0}^{1} \int_{0}^{1}\left[\left(\frac{\partial u}{\partial x}\right)^{2}+\left(\frac{\partial u}{\partial y}\right)^{2}\right] d x d y+\left.\alpha \int_{0}^{1}\left(\frac{\partial u}{\partial x}\right)^{2}\right|_{y=1} d x
$$

is unbounded from below. Indeed, let $u_{k n}=y^{n} \sin \pi k x$, where $n, k$ are integers. We get

$$
E\left(u_{k n}\right)=\frac{(\pi k)^{2}}{4}\left(\alpha+\frac{1}{2 n+1}\right)+\frac{1}{4} \frac{n^{2}}{2 n-1} .
$$

For $\alpha<0$ we find such an $n^{*}$ that

$$
\alpha+\frac{1}{2 n^{*}+1}<0
$$

It is seen that $E\left(u_{k n^{*}}\right) \rightarrow-\infty$ as $k \rightarrow \infty$.

To solve the minimum problem for $J$, we reduce it to the solution of the following integro-differential equation for $u$

$$
\delta J(u) \equiv \int_{V}(\nabla u \cdot \nabla \delta u-f \delta u) d V+\int_{\Omega_{2}}\left(\alpha \frac{\partial u}{\partial s} \frac{\partial \delta u}{\partial s}-\varphi \delta u\right) d \Omega=0
$$

that must be valid for any $\delta u \in C_{0}^{(2)}$. Eq. (2) is applied to the weak setup of the boundary value problem (1). We denote by $H$ the completion of $C_{0}^{(2)}$ with respect to the energy norm

$$
\|u\|_{H}^{2} \equiv \int_{V} \nabla u \cdot \nabla u d V+\int_{\Omega_{2}} \alpha\left(\frac{\partial u}{\partial s}\right)^{2} d \Omega .
$$

The elements of space $H$ belong to Sobolev's space $W^{1,2}(V)$. By the trace theorem, the elements belong to $W^{1 / 2,2}\left(\Omega_{2}\right)$. However the presence of the member $\int_{\Omega_{2}} \alpha(\partial u / \partial s)^{2} d \Omega$ in the norm of $H$ implies higher regularity of $u$ on $\Omega_{2}$, namely $u \in W^{1,2}\left(\Omega_{2}\right)$. So $H$ is not a closed subspace of $W^{1,2}(V)$.

A weak solution to the problem (1) is $u \in H$ satisfying Eq. (2) for any $\delta u \in H$. We underline again the smoothness properties of $u$ on the boundary of $V$ are different from the ones of a weak solution in the classical membrane theory, see $[1,6,13]$. The weak setup of the problem makes sense for some wide classes of loads $f, \varphi$. If the boundary is sufficiently regular and $\alpha>0$ it can be demonstrated both the existence and uniqueness of the weak solution. Note the limit point of the approximations by the finite element method is a weak solution of the problem under consideration.

When the displacements of the membrane boundary are not constrained, that is when $\Omega_{2}=\Omega$, the equilibrium problem has a solution if and only if

$$
\int_{V} f d V+\int_{\Omega} \varphi d \Omega=0
$$


A solution of this problem is uniquely defined up to the constant $c$ that is an analogue to small free motions for a free elastic body.

The goal of this paper is to study the initial-boundary and boundary value problems of linear elasticity for bodies with surface stresses. We will investigate the properties of the spectrum of elastic bodies in this theory. Sect. 2 presents the governing relations of the linear elasticity with regard to surface stresses. Here we used the direct tensor notations as in [12].

The weak setup of equilibrium problems is presented in Sect. 3. We introduce the setup of the problems in energy spaces and prove the theorem of uniqueness and existence of the weak solution in Sect. 4. In Sect. 5 we establish some properties of the eigenvalue problems and apply them to dynamic problems in Sect. 7. Last, but not least, we present the Rayleigh principle for bodies with surface stresses in Sect. 6.

\section{Basic equations of linear elasticity with surface stresses}

From now we change the meaning of the notations of Sect. 1 to three dimensions. For example, $V$ is a bounded domain in $\mathbb{R}^{3}$ with sufficiently regular boundary that a body occupies.

First we consider the problems with mixed boundary conditions. Suppose $\Omega_{1}$, a nonempty part of the boundary surface $\Omega$ of $V$, to be fixed: $\left.\boldsymbol{u}\right|_{\Omega_{1}}=\mathbf{0}$. On the rest part $\Omega_{2}=\Omega \backslash \Omega_{1}$ it is defined the stress vector $\boldsymbol{t}$ expressed through a given load $\varphi$ and $t_{S}$ (the stress vector due the surface stresses) by the formula

$$
t=\varphi+t_{S}
$$

where $t_{S}$ is determined through the surface stress tensor $\tau[4,5,8,9,17]$. So

$$
\begin{aligned}
& \nabla \cdot \boldsymbol{\sigma}+\rho \boldsymbol{f}=\rho \ddot{\boldsymbol{u}}, \quad \boldsymbol{x} \in V, \\
& \left.\boldsymbol{u}\right|_{\Omega_{1}}=\mathbf{0},\left.\quad \boldsymbol{n} \cdot \boldsymbol{\sigma}\right|_{\Omega_{2}}=\boldsymbol{t}, \quad \boldsymbol{x} \in \Omega,
\end{aligned}
$$

where $\sigma$ is the stress tensor, $\nabla$ the 3D gradient operator (3D nabla operator), $\rho$ the body density, $f$ the density of the volume forces, $\boldsymbol{n}$ the external unit normal to $\Omega$, and the dot over a quantity denotes its partial derivative with respect to $t$. The surface stress vector is defined by

$$
\boldsymbol{t}_{S}=\nabla_{S} \cdot \tau,
$$

where $\tau$ is the surface stress tensor on $\Omega$ and $\nabla_{S}$ is the nabla operator on the surface $\Omega$ that relates with $\nabla$ by the formula

$$
\nabla_{S}=\nabla-\boldsymbol{n} \frac{\partial}{\partial z}
$$

and $z$ is the coordinate along the normal to $\Omega$.

In addition, we consider the problem when the static conditions are given on the whole boundary

$$
\left.\boldsymbol{n} \cdot \boldsymbol{\sigma}\right|_{\Omega}=\boldsymbol{t}, \quad \boldsymbol{x} \in \Omega .
$$

For simplicity, we restrict ourselves to an isotropic material. The constitutive equation for the material is the Hooke's law

$$
\boldsymbol{\sigma}=2 \mu \varepsilon+\lambda \mathbf{I} \operatorname{tr} \varepsilon \quad \text { with } \boldsymbol{\varepsilon}=\boldsymbol{\varepsilon}(\boldsymbol{u}) \equiv \frac{1}{2}\left(\nabla \boldsymbol{u}+(\nabla \boldsymbol{u})^{T}\right)
$$

For the surface stresses we assume the following constitutive equation $[4,5]$

$$
\boldsymbol{\tau}=2 \mu_{S} \boldsymbol{\epsilon}+\lambda_{S} \mathbf{A} \operatorname{tr} \boldsymbol{\epsilon} \quad \text { with } \boldsymbol{\epsilon}=\boldsymbol{\epsilon}(\boldsymbol{v}) \equiv \frac{1}{2}\left(\nabla_{S} \boldsymbol{v} \cdot \mathbf{A}+\mathbf{A} \cdot\left(\nabla_{S} \boldsymbol{v}\right)^{T}\right)
$$

where $\boldsymbol{v}$ is the displacement of the film point $\boldsymbol{x}$ of $\Omega_{2}$. Here $\mathbf{I}$ and $\mathbf{A} \equiv \mathbf{I}-\boldsymbol{n} \otimes \boldsymbol{n}$ are the three- and two-dimensional unit tensors, respectively, $\lambda$ and $\mu$ are Lamé's coefficients of the bulk material whereas $\lambda_{S}$ and $\mu_{S}$ are the elastic characteristics of the surface film $\Omega_{2}$ (they are the surface analogues of Lamé's moduli), $\varepsilon$ is the small strain tensor, $\boldsymbol{u}$ is the displacement vector, and $\epsilon$ is the surface strain tensor. Following [8,17], we use the non-separation condition

$$
\left.\boldsymbol{u}\right|_{\Omega_{2}}=\boldsymbol{v}
$$

This explicitly states that the displacements of the surface film $\Omega_{2}$ coincide with the body displacements on the boundary. There are more general relations for the surface stresses that include residual stresses, anisotropy and other factors, cf. $[8,9,17]$. 


\section{Equilibrium problems}

In equilibrium, the dynamic Eq. (3) changes to

$$
\nabla \cdot \boldsymbol{\sigma}+\rho \boldsymbol{f}=\mathbf{0}
$$

Thus the equilibrium boundary value problem for an elastic body with surface stresses consists of Eq. (9) and the boundary conditions

$$
\left.\boldsymbol{u}\right|_{\Omega_{1}}=\mathbf{0},\left.\quad\left(\boldsymbol{n} \cdot \boldsymbol{\sigma}-\nabla_{S} \cdot \boldsymbol{\tau}\right)\right|_{\Omega_{2}}=\boldsymbol{\varphi},
$$

where $\sigma$ and $\tau$ satisfy relations (7) and (8), respectively. In Eq. (8) we set

$$
\boldsymbol{\epsilon}=\left.\boldsymbol{\epsilon}(\boldsymbol{u}) \equiv \frac{1}{2}\left(\nabla_{S} \boldsymbol{u} \cdot \mathbf{A}+\mathbf{A} \cdot\left(\nabla_{S} \boldsymbol{u}\right)^{T}\right)\right|_{\Omega_{2}} .
$$

If $\Omega_{2}=\Omega$ then part $\Omega_{1}$ is absent.

With respect to the constitutive Eqs. (7) and (8) the boundary value problem (9), (10) takes the variational form similar to the Lagrange's principle.

Theorem 3.1. A stationary point $\boldsymbol{u}$ of $J(\boldsymbol{u})=E(\boldsymbol{u})-A(\boldsymbol{u})$ on the set of admissible sufficiently smooth displacements, that is they satisfy the condition $\left.\boldsymbol{u}\right|_{\Omega_{1}}=\mathbf{0}, \Omega_{1} \neq \emptyset$, is a solution of the equilibrium Eqs. (9) for the elastic body in the volume $V$ together with the boundary condition (10), and vice versa. Here

$$
\begin{aligned}
& E(\boldsymbol{u})=\int_{V} W(\boldsymbol{\varepsilon}) d V+\int_{\Omega_{2}} U(\boldsymbol{\epsilon}) d \Omega, \quad A(\boldsymbol{u})=\int_{V} \rho \boldsymbol{f} \cdot \boldsymbol{u} d V+\int_{\Omega_{2}} \boldsymbol{\varphi} \cdot \boldsymbol{u} d \Omega, \\
& W(\boldsymbol{\varepsilon}) \equiv \frac{1}{2} \lambda \operatorname{tr}^{2} \boldsymbol{\varepsilon}+\mu \varepsilon: \boldsymbol{\varepsilon}, \quad U(\boldsymbol{\epsilon}) \equiv \frac{1}{2} \lambda_{S} \operatorname{tr}^{2} \boldsymbol{\epsilon}+\mu_{S} \boldsymbol{\epsilon}: \boldsymbol{\epsilon},
\end{aligned}
$$

where $W$ is the strain energy of the isotropic elastic body, $U$ is the surface strain energy, and : means the scalar product of two second-order tensors, i.e. $\boldsymbol{\alpha}: \boldsymbol{\beta}=\operatorname{tr}\left(\boldsymbol{\alpha} \cdot \boldsymbol{\beta}^{T}\right)$.

Proof. The stationary condition for $J$ leads to the variational equation

$$
\delta J \equiv \int_{V}(\boldsymbol{\sigma}: \delta \boldsymbol{\varepsilon}-\rho \boldsymbol{f} \cdot \delta \boldsymbol{u}) d V+\int_{\Omega_{2}}(\boldsymbol{\tau}: \delta \boldsymbol{\epsilon}-\boldsymbol{\varphi} \cdot \delta \boldsymbol{u}) d \Omega=0
$$

for any sufficiently smooth $\delta \boldsymbol{u}$ such that $\left.\delta \boldsymbol{u}\right|_{\Omega_{1}}=\mathbf{0}$. Applying the Gauss-Ostrogradsky theorem to the integral

$$
\int_{V} \boldsymbol{\sigma}: \delta \varepsilon d V
$$

and noting $\left.\delta \boldsymbol{u}\right|_{\Omega_{1}}=\mathbf{0}$ we get

$$
\begin{aligned}
\int_{V} \boldsymbol{\sigma}: \delta \varepsilon d V & =\int_{V} \boldsymbol{\sigma}:(\nabla \delta \boldsymbol{u})^{T} d V=-\int_{V}(\nabla \cdot \boldsymbol{\sigma}) \cdot \delta \boldsymbol{u} d V+\int_{\Omega} \boldsymbol{n} \cdot \boldsymbol{\sigma} \cdot \delta \boldsymbol{u} d \Omega \\
& =-\int_{V}(\nabla \cdot \boldsymbol{\sigma}) \cdot \delta \boldsymbol{u} d V+\int_{\Omega_{2}} \boldsymbol{n} \cdot \boldsymbol{\sigma} \cdot \delta \boldsymbol{u} d \Omega .
\end{aligned}
$$

Transforming the second integral in (11) together with the surface version of the Gauss-Ostrogradsky theorem [8] we get

$$
\int_{\Omega_{2}} \boldsymbol{\tau}: \delta \boldsymbol{\epsilon} d \Omega=\int_{\Omega_{2}} \boldsymbol{\tau}:\left(\nabla_{S} \delta \boldsymbol{u}\right)^{T} d \Omega=-\int_{\Omega_{2}}\left(\nabla_{S} \cdot \boldsymbol{\tau}\right) \cdot \delta \boldsymbol{u} d \Omega+\int_{\Gamma} \boldsymbol{\nu} \cdot \boldsymbol{\tau} \cdot \delta \boldsymbol{u} d s
$$

where $\boldsymbol{\nu}$ is the external unit normal to the boundary contour $\Gamma$ of $\Omega_{2}, \nu$ lies in the tangent plane to $\Omega$, that is $\boldsymbol{\nu} \cdot \boldsymbol{n}=0$. On $\Omega_{1}$ admissible displacement $\delta \boldsymbol{u}=\mathbf{0}$ so by continuity, $\delta \boldsymbol{u}=\mathbf{0}$ on $\Gamma$ as well. If $\Omega_{2}=\Omega$ the contour integral is absent. Thus the following formula for the first variation of $J$ can be stated

$$
\delta J=-\int_{V}(\nabla \cdot \boldsymbol{\sigma}+\rho \boldsymbol{f}) \cdot \delta \boldsymbol{u} d V+\int_{\Omega_{2}}\left(\boldsymbol{n} \cdot \boldsymbol{\sigma}-\nabla_{S} \cdot \boldsymbol{\tau}-\boldsymbol{\varphi}\right) \cdot \delta \boldsymbol{u} d \Omega .
$$

From (11) we derive Eqs. (9) and (10); when $\Omega_{2}=\Omega$, condition (10) changes to (6).

To prove the second part of the theorem we suppose $\boldsymbol{u}$ to be a solution of the problem (9), (10). Dot-multiplying (9) by $\delta \boldsymbol{u}$ and integrating the results over $V$ we then repeat the above transformations in the reverse order. This brings us to the necessary variational equation $\delta J=0$. 
Suppose $W$ and $U$ to be positive definite functions of their arguments, that is there exist positive constants $c_{1}, c_{2}$ such that

$$
W(\varepsilon) \geq c_{1} \varepsilon: \varepsilon, \quad U(\boldsymbol{\epsilon}) \geq c_{2} \epsilon: \boldsymbol{\epsilon}, \quad c_{1}, c_{2}>0
$$

for any $\varepsilon, \epsilon$. For an isotropic homogeneous material this leads to the inequalities

$$
3 \lambda+2 \mu>0, \quad \mu>0, \quad \lambda_{S}+\mu_{S}>0, \quad \mu_{S}>0 .
$$

We should note that if $U$ can be negative for some deformations then, as for the problem (1), it can be shown that $E$ is not bounded from below that contradicts to the physical meaning of the strain energy.

Let $\boldsymbol{u}^{\circ}$ be a solution of the problem. Then $J(\boldsymbol{u})$ can be represented in the form

$$
J(\boldsymbol{u})=J\left(\boldsymbol{u}-\boldsymbol{u}^{\circ}\right)-J\left(\boldsymbol{u}^{\circ}\right)
$$

From this and the positivity assumptions (13) it follows

Theorem 3.2. Let the assumptions (13) hold and $\Omega_{1} \neq \emptyset$. On the set of admissible smooth displacements $\boldsymbol{u}$ satisfying $\left.\boldsymbol{u}\right|_{\Omega_{1}}=\mathbf{0}$, a stationary point of $J(\boldsymbol{u})$ is a point of minimum of $J(\boldsymbol{u})$.

\section{Weak solutions to the equilibrium problems}

Let us introduce the energy space in which we will seek a weak solution. We start with the set $\boldsymbol{C}_{0}^{(2)}$ of vector functions $\boldsymbol{u}$ that take values in $\mathbb{R}^{3}$ such that each of its Cartesian components belongs to $C^{(2)}(\bar{V})$ and $\left.\boldsymbol{u}\right|_{\Omega_{1}}=\mathbf{0}$. On $\boldsymbol{C}_{0}^{(2)}$ we introduce the inner product

$$
\langle\boldsymbol{u}, \boldsymbol{v}\rangle_{e}=\int_{V}[\lambda \operatorname{tr} \boldsymbol{\varepsilon}(\boldsymbol{u}) \operatorname{tr} \boldsymbol{\varepsilon}(\boldsymbol{v})+2 \mu \varepsilon(\boldsymbol{u}): \boldsymbol{\varepsilon}(\boldsymbol{v})] d V+\int_{\Omega_{2}}\left[\lambda_{S} \operatorname{tr} \boldsymbol{\epsilon}(\boldsymbol{u}) \operatorname{tr} \boldsymbol{\epsilon}(\boldsymbol{v})+2 \mu_{S} \boldsymbol{\epsilon}(\boldsymbol{u}): \boldsymbol{\epsilon}(\boldsymbol{v})\right] d \Omega
$$

and the corresponding energy norm $\|\boldsymbol{u}\|_{e}^{2}=\langle\boldsymbol{u}, \boldsymbol{u}\rangle_{e}$ that is the double strain energy of the body $2 E(\boldsymbol{u})$. Suppose the conditions (13) are fulfilled. Then on $\boldsymbol{C}_{0}^{(2)}$ form $\langle\boldsymbol{u}, \boldsymbol{v}\rangle_{e}$ possesses all the properties of the inner product. However $\boldsymbol{C}_{0}^{(2)}$ with this inner product is not complete.

Definition 4.1. The completion of $\boldsymbol{C}_{0}^{(2)}$ with respect to the norm $\|\boldsymbol{u}\|_{e}$ is called the energy space $\boldsymbol{E}$.

By Korn's inequality on $C_{0}^{(2)}$, cf. for example [1,6,11], it is easily seen that

$$
\|\boldsymbol{u}\|_{W^{1,2}(V)} \leq C_{1}\|\boldsymbol{u}\|_{e}
$$

with some constant $C_{1}$ that does not depend on $\boldsymbol{u} \in \boldsymbol{E}$. To prove this inequality, we should suppose some regularity of the boundary of $V$, for example, it should satisfy the cone property $[1,6,11,13,14]$. So we can use the properties of the Sobolev space $W^{1,2}(V)$ for the elements of space $\boldsymbol{E}$. Note that the Sobolev norm for the vector functions on the domain $V$ is defined by the formula

$$
\|\boldsymbol{u}\|_{W^{1,2}(V)}^{2}=\int_{V}\left(|\boldsymbol{u}|^{2}+\nabla \boldsymbol{u}: \nabla \boldsymbol{u}^{T}\right) d V
$$

Let $\Omega_{2}$ consists of a finite number of sufficiently smooth surfaces with smooth boundaries. Using the techniques of the shell theory, cf. [2,19], it is easy to prove an analogue of Korn's inequality on $\Omega_{2}$,

$$
\|\boldsymbol{u}\|_{W^{1,2}\left(\Omega_{2}\right)} \leq C_{2}\|\boldsymbol{u}\|_{e}
$$

with some constant $C_{2}$ that does not depend on $\boldsymbol{u} \in \boldsymbol{E}$. Here

$$
\|\boldsymbol{u}\|_{W^{1,2}\left(\Omega_{2}\right)}^{2}=\int_{\Omega_{2}}\left(|\boldsymbol{u}|^{2}+\nabla_{S} \boldsymbol{u}: \nabla_{S} \boldsymbol{u}^{T}\right) d \Omega .
$$

Again as for the membrane, $\boldsymbol{u}$ of $\boldsymbol{E}$ is an element of $W^{1,2}(V)^{3}$ and so by the trace theorem, it belongs to $W^{1 / 2,2}\left(\Omega_{2}\right)^{3}$. However the presence of the member given on $\Omega_{2}$ in the norm of $\boldsymbol{E}$ implies that $\boldsymbol{u} \in \boldsymbol{E}$ possesses higher regularity, namely $\boldsymbol{u} \in W^{1,2}\left(\Omega_{2}\right)^{3}$. 
Now we introduce:

Definition 4.2. A weak solution of the problem (9), (10) is $\boldsymbol{u} \in \boldsymbol{E}$ that satisfies Eq. (11) for any $\delta \boldsymbol{u} \in \boldsymbol{E}$.

Let us formulate:

Theorem 4.3. Let $f \in L^{6 / 5}(V)^{3}$ and $\varphi \in L^{p}\left(\Omega_{2}\right)^{3}$ for some $p>1$. There exists a weak solution of the problem (9), (10) by Definition 4.2 that is unique.

Proof. By the properties of the Sobolev space $W^{1,2}$, functional $A(\delta \boldsymbol{u})$ is linear and continuous in $\boldsymbol{E}$; it can be proved in a similar manner to the proof of this fact for the work functional in linear elasticity, cf. [1, 6, 13]. By the Riesz representation theorem for a linear continuous functional in a Hilbert space we have

$$
A(\delta \boldsymbol{u})=\left\langle\boldsymbol{u}_{0}, \delta \boldsymbol{u}\right\rangle_{e},
$$

where $\boldsymbol{u}_{0}$ is a uniquely defined element of $\boldsymbol{E}$. So Eq. (11) reduces to the equation

$$
\langle\boldsymbol{u}, \delta \boldsymbol{u}\rangle_{e}=\left\langle\boldsymbol{u}_{0}, \delta \boldsymbol{u}\right\rangle_{e}
$$

for any $\delta \boldsymbol{u} \in \boldsymbol{E}$. This equation has the unique solution $\boldsymbol{u}=\boldsymbol{u}_{0}$ that completes the proof.

Using the techniques for the equilibrium problem for a free body under load in the classic linear elasticity [11, 13], we can prove the following theorem for a body free of geometrical constraints. Now $\Omega_{2}=\Omega$.

Theorem 4.4. Let $f \in L^{6 / 5}(V)^{3}$ and $\varphi \in L^{p}\left(\Omega_{2}\right)^{3}$ for some $p>1$ and the external forces be self-balanced, that is

$$
\int_{V} \boldsymbol{f} d V+\int_{\Omega} \boldsymbol{\varphi} d \Omega=\mathbf{0}, \quad \int_{V} \boldsymbol{x} \times \boldsymbol{f} d V+\int_{\Omega} \boldsymbol{x} \times \boldsymbol{\varphi} d \Omega=\mathbf{0} .
$$

There exists a weak solution of the problem (9), (10) by Definition 4.2 that is unique up to the infinitesimal rigid body motions $\boldsymbol{a}+\boldsymbol{x} \times \boldsymbol{b}$, where $\boldsymbol{a}, \boldsymbol{b}$ are constant vectors and $\boldsymbol{x}$ is the position vector of a body point.

To solve the equilibrium of solids with surface stresses they use the method of finite elements. We briefly discuss some features of the conforming version of the method [3]. In this version we generate the sequence of the finite-dimensional subspaces $\boldsymbol{E}_{h}$ of the space $E$ and find the approximate FEM solutions $\boldsymbol{u}_{h} \in \boldsymbol{E}_{h}$. By Céa's lemma [3] we have the bound for the error

$$
\left\|\boldsymbol{u}-\boldsymbol{u}_{h}\right\|_{e} \leq C \inf _{\boldsymbol{u}_{h} \in \boldsymbol{E}_{h}}\left\|\boldsymbol{u}-\boldsymbol{u}_{h}\right\|_{e}
$$

where $\boldsymbol{u} \in \boldsymbol{E}$ is the weak solution, and $C$ a constant. This estimate depends on the error of the approximation in the volume and on the approximation error on the surface $\Omega_{2}$.

\section{Eigenvalue problems in the theory of elasticity with surface stresses}

Let us consider an important case of dynamical problems: the eigenoscillation problem for an elastic body with surface stresses. In this problem we seek solutions to the homogeneous dynamic equation in displacements $(f=0, \varphi=0)$ in the following form:

$$
\boldsymbol{u}=\boldsymbol{u}(\boldsymbol{x}, t)=\boldsymbol{w}(\boldsymbol{x}) \mathrm{e}^{i \omega t} .
$$

Substituting this into (3), (10) expressed in displacements and canceling the factor $\mathrm{e}^{i \omega t}$, we get

$$
\nabla \cdot \boldsymbol{\sigma}=-\rho \omega^{2} \boldsymbol{w} \quad \text { in } V,\left.\quad \boldsymbol{w}\right|_{\Omega_{1}}=\mathbf{0},\left.\quad\left(\boldsymbol{n} \cdot \boldsymbol{\sigma}-\nabla_{S} \cdot \boldsymbol{\tau}\right)\right|_{\Omega_{2}}=\mathbf{0} .
$$

In the last Eqs. $\boldsymbol{\sigma}$ and $\boldsymbol{\tau}$ are given by the relations (7) and (8) with $\varepsilon=\varepsilon(\boldsymbol{w}), \boldsymbol{\epsilon}=\boldsymbol{\epsilon}(\boldsymbol{w})$.

Eqs. (16) constitute an eigenvalue problem: we should find positive values $\omega$, called eigenfrequencies of the elastic body with surface stresses, for which problem (16) has a non-trivial solution $\boldsymbol{w}$ that is called an eigenoscillation. A normed $\boldsymbol{w}$ will be called the oscillation eigenmode. Let us note that for the boundary value problems with boundary condition (10), the minimal eigenfrequency is positive. Indeed, for $\omega=0$ the problem (16) is described by the equilibrium equations, and by the uniqueness of the solution of this equilibrium problem we have the only solution $\boldsymbol{w}=\mathbf{0}$. For the second boundary value problem with condition (6) that is when $\Omega_{2}=\Omega$, there corresponds to the "frequency" $\omega=0$ a non-trivial solution that represents infinitesimal translations and rotations of a rigid body

$$
\boldsymbol{w}=\boldsymbol{a}+\boldsymbol{b} \times \boldsymbol{x}
$$

with arbitrary but constant vectors $\boldsymbol{a}$ and $\boldsymbol{b}$. Let us restrict ourselves to the case of positive eigenfrequencies $\omega$.

We present the following theorem. 
Theorem 5.1. For the oscillation eigenmodes $\boldsymbol{w}_{1}$ and $\boldsymbol{w}_{2}$ corresponding to distinct eigenfrequencies $\omega_{1}$ and $\omega_{2}$, respectively, the relation

$$
\int_{V} \rho \boldsymbol{w}_{1} \cdot \boldsymbol{w}_{2} d V=0
$$

holds true. Moreover,

$$
\left\langle\boldsymbol{w}_{1}, \boldsymbol{w}_{2}\right\rangle_{e}=0
$$

Eq. (17) is called the orthogonality relation, and (18) - the generalized orthogonality relation for $\boldsymbol{w}_{1}$ and $\boldsymbol{w}_{2}$.

Proof. Let eigenmodes $\boldsymbol{w}_{1}$ and $\boldsymbol{w}_{2}$ correspond to different eigenfrequencies, so that

$$
\nabla \cdot \boldsymbol{\sigma}_{1}=-\rho \omega_{1}^{2} \boldsymbol{w}_{1} \quad \text { in } V,\left.\quad \boldsymbol{w}_{1}\right|_{\Omega_{1}}=\mathbf{0},\left.\quad \boldsymbol{n} \cdot \boldsymbol{\sigma}_{1}\right|_{\Omega_{2}}=\nabla_{S} \cdot \boldsymbol{\tau}_{1}
$$

and

$$
\nabla \cdot \boldsymbol{\sigma}_{2}=-\rho \omega_{2}^{2} \boldsymbol{w}_{2} \quad \text { in } V,\left.\quad \boldsymbol{w}_{2}\right|_{\Omega_{1}}=\mathbf{0},\left.\quad \boldsymbol{n} \cdot \boldsymbol{\sigma}_{2}\right|_{\Omega_{2}}=\nabla_{S} \cdot \boldsymbol{\tau}_{2},
$$

where $\boldsymbol{\sigma}_{k}$ and $\boldsymbol{\tau}_{k}$ are defined by (7) and (8). Denote $\boldsymbol{\varepsilon}_{k}=\boldsymbol{\varepsilon}\left(\boldsymbol{w}_{k}\right)$ and $\boldsymbol{\epsilon}_{k}=\boldsymbol{\epsilon}\left(\boldsymbol{w}_{k}\right)$. Dot-multiply the first equation given on $V$ by $\boldsymbol{w}_{2}$ and integrate over $V$. Applying the Gauss-Ostrogradsky theorem we get

$$
-\int_{V} \boldsymbol{\sigma}_{1}: \varepsilon_{2} d V-\int_{\Omega_{2}} \boldsymbol{\tau}_{1}: \boldsymbol{\epsilon}_{2} d \Omega+\omega_{1}^{2} \int_{V} \rho \boldsymbol{w}_{1} \cdot \boldsymbol{w}_{2} d V=0 .
$$

Similarly, one obtains

$$
-\int_{V} \boldsymbol{\sigma}_{2}: \varepsilon_{1} d V-\int_{\Omega_{2}} \boldsymbol{\tau}_{2}: \boldsymbol{\epsilon}_{2} d \Omega+\omega_{2}^{2} \int_{V} \rho \boldsymbol{w}_{2} \cdot \boldsymbol{w}_{1} d V=0 .
$$

Subtract these two equations. Since $\sigma_{1}: \varepsilon_{2}=\sigma_{2}: \varepsilon_{1}$ and $\tau_{1}: \epsilon_{2}=\tau_{2}: \epsilon_{1}$, we get

$$
\left(\omega_{1}^{2}-\omega_{2}^{2}\right) \int_{V} \rho \boldsymbol{w}_{1} \cdot \boldsymbol{w}_{2} d V=0 .
$$

$\omega_{1} \neq \omega_{2}$, so the relation (17) follows immediately. Substituting (17) into (19) we obtain (18).

Now we represent Eq. (16) in the weak form

$$
\langle\boldsymbol{w}, \boldsymbol{v}\rangle_{e}=\rho \omega^{2} \int_{V} \boldsymbol{w} \cdot \boldsymbol{v} d V
$$

that must hold for any $\boldsymbol{v} \in \boldsymbol{E}$. We seek a weak solution of this so $\boldsymbol{w} \in \boldsymbol{E}$. Let us transform Eq. (20) to an operator equation. For any fixed $\boldsymbol{w} \in \boldsymbol{E}$ the functional $\rho \omega^{2} \int_{V} \boldsymbol{w} \cdot \boldsymbol{v} d V$ is linear and continuous in $\boldsymbol{v} \in \boldsymbol{E}$. By the Riesz representation theorem for a linear continuous functional in a Hilbert space there is an unique element $\boldsymbol{w}_{0} \in \boldsymbol{E}$ such that

$$
\int_{V} \boldsymbol{w} \cdot \boldsymbol{v} d V=\left\langle\boldsymbol{w}_{0}, \boldsymbol{v}\right\rangle_{e}
$$

for any $\boldsymbol{v} \in \boldsymbol{E}$. The correspondence $\boldsymbol{w}$ to $\boldsymbol{w}_{0}$ defines a linear operator $\boldsymbol{w}_{0}=B \boldsymbol{w}$. This operator possesses the properties of the analogous operator in the classical linear elasticity, that is it is linear, selfadjoint, compact and positive definite, cf. $[1,6,13]$. By a general theorem of functional analysis, we now can formulate other properties of the spectrum and eigensolutions of the problem under consideration, cf. $[1,6,13]$. We present it as:

Theorem 5.2. The eigenvalue problem under consideration has a discrete spectrum which contains only eigenfrequencies $\omega_{k}$ that possess the following properties

- All $\omega_{k}$ are positive, $\omega_{k} \geq \omega>0$.

- The set $\left\{\omega_{k}\right\}$ is infinite and does not contain a finite limit point.

- To each $\omega_{k}$ there corresponds no more than a finite number of linearly independent eigensolutions which are assumed to be orthonormalized.

- The set of all these eigenmodes $\left\{\boldsymbol{w}_{k}\right\}$ is a complete orthonormal system in the energy space $\boldsymbol{E}$. Besides the set $\left\{\omega_{k} \boldsymbol{w}_{k}\right\}$ is a complete orthonormal system in $L^{2}(V)$ with the scalar product

$$
(\boldsymbol{w}, \boldsymbol{v})=\int_{V} \rho \boldsymbol{w} \cdot \boldsymbol{v} d V
$$




\section{Rayleigh variational principle}

On the orthogonality of the eigensolutions established in Theorem 5.1 practical applications of the Rayleigh variational principle can be grounded.

Theorem 6.1. Eigenmodes are stationary points of the energy functional

$$
E(\boldsymbol{w})=\int_{V} W(\varepsilon(\boldsymbol{w})) d V+\int_{\Omega_{2}} U(\boldsymbol{\epsilon}(\boldsymbol{w})) d \Omega
$$

on the set of displacements satisfying the boundary conditions $\left.\boldsymbol{w}\right|_{\Omega_{1}}=\mathbf{0}$ and subject to the constraint

$$
\frac{1}{2} \int_{V} \rho \boldsymbol{w} \cdot \boldsymbol{w} d V=1
$$

Conversely, all the stationary points of $E(\boldsymbol{w})$ on the above set of displacements are eigenmodes of the body that correspond to its eigenfrequencies.

Proof. Let us write down the stationarity condition for $E(\boldsymbol{w})$. Using the same reasoning as in the Theorem 3.1, we get

$$
\delta E=-\int_{V}(\nabla \cdot \boldsymbol{\sigma}) \cdot \delta \boldsymbol{w} d V+\int_{\Omega_{2}}\left(\boldsymbol{n} \cdot \boldsymbol{\sigma}-\nabla_{S} \cdot \boldsymbol{\tau}\right) \cdot \delta \boldsymbol{w} d \Omega=0
$$

Using the Lagrange multipliers method, the stationarity of $E$ implies two simultaneous equations

$$
\delta E-\ell \int_{V} \rho \boldsymbol{w} \cdot \delta \boldsymbol{w} d V=0, \quad \frac{1}{2} \int_{V} \rho \boldsymbol{w} \cdot \boldsymbol{w} d V-1=0 .
$$

From the first equation in (23) we get

$$
-\int_{V}[(\nabla \cdot \boldsymbol{\sigma}) \cdot \delta \boldsymbol{w}+\ell \rho \boldsymbol{w} \cdot \delta \boldsymbol{w}] d V+\int_{\Omega_{2}}\left(\boldsymbol{n} \cdot \boldsymbol{\sigma}-\nabla_{S} \cdot \boldsymbol{\tau}\right) \cdot \delta \boldsymbol{w} d \Omega
$$

It follows from this integral equation for arbitrary $\delta \boldsymbol{w}$ that

$$
\nabla \cdot \boldsymbol{\sigma}=-\rho \ell \boldsymbol{w},\left.\quad \boldsymbol{n} \cdot \boldsymbol{\sigma}\right|_{\Omega_{2}}=\nabla_{S} \cdot \boldsymbol{\tau}
$$

If we change $\ell$ to $\omega^{2}$, these equations coincides with (16). Hence, $\ell$ is equal to the squared eigenvalue $\omega$. Thus the stationarity condition for $E(\boldsymbol{w})$ is valid on eigensolutions of the problem (16).

We omit the proof of the converse statement of the theorem as we should only repeat the proof above in the reverse mode.

We will change the form of Rayleigh's principle to the one frequently used in applications. In the new formulation, one needs not stipulate separate integral restrictions on the set of $\boldsymbol{w}$.

Theorem 6.2. On the set of admissible vector-functions satisfying the condition $\left.\boldsymbol{w}\right|_{\Omega_{1}}=\mathbf{0}$, the oscillation eigenmodes are stationary points of the functional

$$
R(\boldsymbol{w})=\frac{E(\boldsymbol{w})}{K(\boldsymbol{w})} \quad \text { where } \quad K(\boldsymbol{w})=\frac{1}{2} \int_{V} \rho \boldsymbol{w} \cdot \boldsymbol{w} d V .
$$

$R(\boldsymbol{w})$ is called Rayleigh's quotient. Conversely, a stationary point of $R(\boldsymbol{w})$ is an eigenmode that corresponds to some eigenfrequency; the value of $R(\boldsymbol{w})$ on an eigenmode is the squared eigenfrequency

$$
R(\boldsymbol{w})=\omega^{2} .
$$

Property (25) allows to estimate the eigenvalues using some approximation for the eigenmodes. 


\section{On dynamic problems in elasticity with surface stresses}

In the dynamic theory under consideration the initial boundary value problem is given by the equations

$$
\begin{array}{r}
\nabla \cdot \boldsymbol{\sigma}+\rho \boldsymbol{f}=\rho \ddot{\boldsymbol{u}} \quad \text { in } V, \\
\left.\boldsymbol{u}\right|_{\Omega_{1}}=\mathbf{0},\left.\quad\left(\boldsymbol{n} \cdot \boldsymbol{\sigma}-\nabla_{S} \cdot \boldsymbol{\tau}\right)\right|_{\Omega_{2}}=\mathbf{0}, \\
\left.\boldsymbol{u}\right|_{t=0}=\boldsymbol{u}_{0},\left.\quad \dot{\boldsymbol{u}}\right|_{t=0}=\boldsymbol{v}_{0},
\end{array}
$$

and relations (7) and (8), where $\boldsymbol{u}_{0}$ and $\boldsymbol{v}_{0}$ are given initial values for the displacements of the points of $V$ and their velocities. Here we consider the weak setup of the problem as well. It differs from the static statement as the displacements depend on time $t$.

To derive the necessary integro-differential equation on the time interval $[0, T]$ we dot-multiply Eq. (26) by the "virtual" sufficiently smooth displacement $\delta \boldsymbol{u}$ that satisfies the condition $\left.\delta \boldsymbol{u}\right|_{\Omega_{1}}=\mathbf{0}$ and besides $\left.\delta \boldsymbol{u}\right|_{t=T}=\mathbf{0}$. Integrating the result over $V$ and repeating the transformations done for the equilibrium problem we get

$$
\int_{V}(\boldsymbol{\sigma}: \delta \boldsymbol{\varepsilon}-\rho \boldsymbol{f} \cdot \delta \boldsymbol{u}) d V+\int_{\Omega_{2}} \boldsymbol{\tau}: \delta \boldsymbol{\epsilon} d \Omega=-\int_{V} \rho \ddot{\boldsymbol{u}} \cdot \delta \boldsymbol{u} d V .
$$

Now we integrate this with respect to $t$ over $[0, T]$ and then integrate by parts in the term containing $\ddot{\boldsymbol{u}}$. Using $\left.\delta \boldsymbol{u}\right|_{t=T}=\mathbf{0}$ we get the following equation

$$
\int_{0}^{T}\langle\boldsymbol{u}, \delta \boldsymbol{u}\rangle_{e} d t=\int_{0}^{T} \int_{V} \rho \boldsymbol{f} \cdot \delta \boldsymbol{u} d V d t+\int_{0}^{T} \int_{V} \rho \dot{\boldsymbol{u}} \cdot \delta \dot{\boldsymbol{u}} d V d t+\int_{V} \rho \boldsymbol{v}_{0} \cdot \delta \boldsymbol{u} d V
$$

that is used for the weak formulation of the problem.

Let us introduce the energy space that is appropriate for the weak setup.

Definition 7.1. $\boldsymbol{E}(0, T)$ is the completion of the set of all the vector functions $\boldsymbol{u}(\boldsymbol{r}, t) \in\left(C^{(2)}(V \times[0, T])\right)^{3}$ that vanish on $\Omega_{1}$ with respect to the norm induced by the energy scalar product

$$
\langle\boldsymbol{u}, \delta \boldsymbol{u}\rangle_{\boldsymbol{E}_{(0, T)}}=\int_{0}^{T}\langle\boldsymbol{u}, \delta \boldsymbol{u}\rangle_{e} d t+\int_{0}^{T} \int_{V} \rho \dot{\boldsymbol{u}} \cdot \dot{\delta} \boldsymbol{u} d V d t
$$

$D_{0}^{T}$ is the subspace of $\boldsymbol{E}(0, T)$ that is the result of the completion of the subset $\left(C^{(2)}(V \times[0, T])\right)^{3}$ of the vector functions that are zero on $\Omega_{1}$ and take zero value at $t=T$.

The set of the elements of $\boldsymbol{E}(0, T)$ can be considered as a subspace of $\left(W^{(1,2)}(V \times[0, T])\right)^{3}$ with some additional properties on the surface $\Omega_{2}$, cf. [1,6,11] so we can use the properties of this Sobolev space [14].

Now we introduce:

Definition 7.2. $\boldsymbol{u} \in \boldsymbol{E}(0, T)$ is called a generalized solution of the dynamic problem of the body with surface stresses if it satisfies Eq. (28) with any $\delta \boldsymbol{u} \in D_{0}^{T}$ and the initial condition $\left.\boldsymbol{u}\right|_{t=0}=\boldsymbol{u}_{0}$ in the sense of $L^{2}(V)$, that is

$$
\int_{V}\left|\boldsymbol{u}(\boldsymbol{r}, 0)-\boldsymbol{u}_{0}(\boldsymbol{r})\right|^{2} d V=0 .
$$

Note the condition for the initial velocity enters to Eq. (28).

Theorem 7.3. Suppose that

- $\boldsymbol{u}_{0} \in \boldsymbol{E}$ and $\boldsymbol{u}_{0}=\mathbf{0}$ on $\Omega_{1}$,

- $\boldsymbol{v}_{0} \in\left(L^{2}(V)\right)^{3}$,

- $\boldsymbol{f} \in\left(L^{2}(V \times[0, T])\right)^{3}$.

There exists (in the sense of Definition 7.2), a generalized solution to the dynamic problem for the elastic body with surface stresses, and it is unique.

We only sketch the proof as it mimics the proof for the dynamic problem for a clamped membrane in [11] (see also [14]). First we apply the Faedo-Galerkin method to find an approximate solution of the problem. We seek an approximation solution in the form

$$
\boldsymbol{u}_{n}(\boldsymbol{r}, t)=\sum_{k=1}^{n} c_{k}(t) \boldsymbol{\varphi}_{k}
$$


where $\left\{\boldsymbol{\varphi}_{k}\right\}$ is a complete system in the space $\boldsymbol{E}$. In particular, for $\left\{\boldsymbol{\varphi}_{k}\right\}$ we can use the orthonormal system of the eigensolutions $\left\{\boldsymbol{w}_{k}\right\}$ from Theorem 5.2. Next, using Eq. (27) we construct a linear system of ordinary differential equations for unknown coefficients $c_{k}$. The equations are of the second order. Using the orthonormal basis $\left\{\boldsymbol{w}_{k}\right\}$, the system splits into $n$ separate equations. Supplying $c_{k}$ with initial values, we prove the initial value system has a solution. Next we should establish an a priory estimate for the approximated solution $\boldsymbol{u}_{n}$ that follows from the energy inequalities and prove that the limit point of the sequence $\left\{\boldsymbol{u}_{n}\right\}$ is a generalized solution. Its uniqueness is demonstrated separately, as it is done in [11,14].

\section{Conclusions}

The mathematical properties of initial and boundary value problems of linear elasticity with surface stresses are presented and discussed. The theorems of existence and uniqueness of the solutions of the static and dynamic problems in energy spaces are formulated and proven. Some properties of the spectrum of the problems are derived. Solutions of the problems under consideration are more smooth on the boundary surface than solutions of corresponding problems of the classical linear elasticity. The considered weak statements of the initial and boundary problems constitute the mathematical foundation for some numerical methods in particular, for the finite element method.

Acknowledgements The second author was supported by the RFBR with the grant No. 09-01-00459 and by the DFG grant No. AL 341/31-1. The third author is supported by the project No. 1123 of National University of Colombia.

\section{References}

[1] P. G. Ciarlet, Mathematical Elasticity. Vol. I: Three-Dimensional Elasticity (North-Holland, Amsterdam, 1988).

[2] P. G. Ciarlet, Mathematical Elasticity. Vol. III: Theory of Shells (North-Holland, Amsterdam, 2000).

[3] P. G. Ciarlet, The Finite Element Method for Elliptic Problems (SIAM, Philadelphia, 2002).

[4] H. L. Duan, J. Wang, Z.P. Huang, and B. L. Karihaloo, Size-dependent effective elastic constants of solids containing nanoinhomogeneities with interface stress, J. Mech. Phys. Solids 53(7), 1574-1596 (2005).

[5] H. L. Duan, J. Wang, and B. L. Karihaloo, Theory of Elasticity at the Nanoscale, in: Advances in Applied Mechanics, (Elsevier, Amsterdam, 2008), pp. 1-68.

[6] G. Fichera, Existence Theorems in Elasticity, in: Handbuch der Physik, Vol. VIa/2, edited by S. Flügge (Springer, Berlin, 1972), pp. 347-389.

[7] J.W. Gibbs, On the Equilibrium of Heterogeneous Substances, in: The Collected Works of J. Willard Gibbs, (Longmans, Green \& Co, New York, 1928), pp. 55-353.

[8] M.E. Gurtin and A.I. Murdoch, A continuum theory of elastic material surfaces, Arch. Ration. Mech. Anal. 57(4), 291-323 (1975).

[9] M. E. Gurtin and A. I. Murdoch, Addenda to our paper A continuum theory of elastic material surfaces, Arch. Ration. Mech. Anal. 59(4), 389-390 (1975).

[10] L.D. Landau and E. M. Lifshitz, Fluid Mechanics, 2nd edition, Course of Theoretical Physics, Vol. 6 (Pergamon Press, Oxford, 1987).

[11] L. P. Lebedev and M. J. Cloud, The Calculus of Variations and Functional Analysis with Optimal Control and Applications in Mechanics (World Scientific, Singapore, 2003).

[12] L. P. Lebedev, M. J. Cloud, and V. A. Eremeyev, Tensor Analysis, 2nd edition (World Scientific, Singapore, to be published).

[13] L. P. Lebedev and I. I. Vorovich, Functional Analysis in Mechanics (Springer, New York, 2002).

[14] J.-L. Lions and E. Magenes, Problèmes aux Limites non Homogènes et Applications (Dunod, Paris, 1968).

[15] P. Podio-Guidugli and G. V. Caffarelli, Surface interaction potentials in elasticity, Arch. Ration. Mech. Anal. 109(4), 343-383 (1990).

[16] Ya. S. Podstrigach and Ya.Z. Povstenko, Introduction to Mechanics of Surface Phenomena in Deformable Solids (in Russian) (Naukova Dumka, Kiev, 1985).

[17] Ya.Z. Povstenko, Theoretical investigation of phenomena caused by heterogeneous surface tension in solids, J. Mech. Phys. Solids 41(9), 1499-1514 (1993).

[18] D. J. Steigmann and R. W. Ogden, Elastic surface-substrate interactions, Proc. R. Soc. Lond. A, Math. Phys. Eng. Sci. 455, $437-$ 474 (1982). (1999).

[19] I. I. Vorovich, Nonlinear Theory of Shallow Shells (Springer, New York, 1999). 